Quant um and pl asma screeni ng effects on the Wanni er threshol d I aw for the doubl e- el ectron escape in strongl y coupl ed semicl assi cal $\mathrm{pl}$ asmas

\begin{tabular}{|c|c|}
\hline $\begin{array}{l}\text { j our nal or } \\
\text { publ i cat } i \text { on } \mathrm{title}\end{array}$ & Physi cs of $\mathrm{Pl}$ asmas \\
\hline vol une & Vol .15 \\
\hline number & I ssue11 \\
\hline page $r$ ange & pp. 114501- 1 - 114501- 4 \\
\hline year & $2008-11-01$ \\
\hline URL & ht t p: //hdl . handl e. net /10655/2197 \\
\hline
\end{tabular}




\title{
Quantum and plasma screening effects on the Wannier threshold law for the double-electron escape in strongly coupled semiclassical plasmas
}

\author{
Young-Dae Jung ${ }^{1,2, a)}$ and Daiji Kato ${ }^{1}$ \\ ${ }^{1}$ National Institute for Fusion Science, Toki, Gifu 509-5292, Japan \\ ${ }^{2}$ Department of Applied Physics, Hanyang University, Ansan, Kyunggi-Do 426-791, South Korea
}

(Received 10 October 2008; accepted 17 October 2008; published online 18 November 2008)

\begin{abstract}
The quantum and plasma screening effects on the Wannier threshold law for the double-electron escape are investigated in strongly coupled semiclassical plasmas. The renormalized electron charge and Wannier exponent are obtained by considering the equation of motion in the Wannier configuration with the screened pseudopotential model as functions of the de Broglie wavelength, Debye length, and charge of the residual ion. It is shown that the renormalized electron charge significantly increases with an increase in the de Broglie wavelength, especially for small radial distances. It is also shown that the quantum effects enhance the Wannier exponent for the double-electron escape. In addition, the quantum effect on the Wannier threshold law is found to be more important than the plasma screening effect in strongly coupled semiclassical plasmas. (C) 2008 American Institute of Physics. [DOI: 10.1063/1.3021456]
\end{abstract}

After the seminal work of Wannier ${ }^{1}$ on the ionization threshold law in 1953, the double-electron escape ${ }^{2,3}$ from an atom or ion has received considerable attention because the investigation of the long-range radial electron-electron correlations has played an important role in many areas of physics. In addition, recently the electron ionization processes ${ }^{4-7}$ in plasmas such as the electron-impact ionization and photoionization have received much attention since these processes provide useful information on various plasma parameters as well as knowledge about the dynamics and structure of atomic systems. It has been shown that the screened particle interaction in weakly coupled plasmas would be characterized mostly by the standard Debye-Hückel model. ${ }^{8,9}$ In these weakly coupled plasmas, the average energy of interaction between plasma particles is known to be smaller than the average kinetic energy of a particle in plasmas. However, it is obvious that the physical processes in such strongly coupled plasmas would be quite different from those in weakly coupled classical plasmas. Moreover, the interest in strongly coupled plasmas has remarkably increased due to recent investigations on various physical properties of astrophysical compact objects and laser induced inertial confinement fusion plasmas. In addition, it has been shown that the interaction potential in strongly coupled semiclassical plasmas would not be represented by the conventional DebyeHückel model because of nonideal particle interactions due to collective and quantum effects. ${ }^{10-13}$ However, the behavior of the Wannier threshold law for the double-electron escape in strongly coupled semiclassical plasmas has not been specifically investigated as yet. Thus, in this brief communication we investigate the quantum and plasma screening effects on the Wannier threshold law for the double-electron escape from the ion in strongly coupled semiclassical plasmas. The

\footnotetext{
${ }^{a}$ Permanent address: Department of Applied Physics, Hanyang University, Ansan, Kyunggi-Do 426-791, South Korea. Electronic mail: ydjung@hanyang.ac.kr.
}

screened renormalized electron is obtained by using the screened pseudopotential model, ${ }^{10}$ taking into account the quantum and correlation effects as a function of the de Broglie wavelength, Debye length, and charge of the residual ion. Furthermore, the quantum and plasma screening effects on the Wannier exponent for the double-electron escape is obtained by considering the screened effective charge of the residual ion in strongly coupled semiclassical plasmas.

The force equation for a system of two electrons $\left(\mathbf{r}_{1}, \mathbf{r}_{2}\right)$ in the field of the ion with nuclear charge $Z e$ is represented by

$$
\mathbf{F}\left(\mathbf{r}_{1}\right)=-\frac{\partial}{\partial \mathbf{r}_{1}}\left[V_{e i}\left(\mathbf{r}_{1}\right)+V_{e e}\left(\mathbf{r}_{1}, \mathbf{r}_{2}\right)\right]
$$

where $\mathbf{F}\left(\mathbf{r}_{1}\right)$ is the force acting on the electron at the position $\mathbf{r}_{1}$ and $V_{e i}\left(\mathbf{r}_{1}\right)$ and $V_{e e}\left(\mathbf{r}_{1}, \mathbf{r}_{2}\right)$ are, respectively, the electronion and electron-electron interaction potentials. Recently, the useful analytic effective pseudopotential ${ }^{10}$ of the particle interactions in strongly coupled semiclassical plasmas has been obtained on the basis of the dielectric response function formalism taking into account the correlation between the Boltzmann factor and quantum mechanical Slater sum. Using the effective pseudopotential model, ${ }^{10}$ the screened electron-ion $V_{e i}\left(\mathbf{r}_{1}\right)$ and electron-electron $V_{e e}\left(\mathbf{r}_{1}, \mathbf{r}_{2}\right)$ interaction potentials in strongly coupled semiclassical plasmas would be obtained by

$$
\begin{aligned}
V_{e i}\left(\mathbf{r}_{1}\right)= & -\frac{Z e^{2}}{\sqrt{1-4 \lambda_{e i}^{2} / r_{D}^{2}}} \frac{1}{r_{1}}\left\{\exp \left[A_{e i}\left(\lambda_{e i}, r_{D}\right) r_{1}\right]\right. \\
& \left.-\exp \left[B_{e i}\left(\lambda_{e i}, r_{D}\right) r_{1}\right]\right\}, \\
V_{e e}\left(\mathbf{r}_{1}, \mathbf{r}_{2}\right)= & \frac{e^{2}}{\sqrt{1-4 \lambda_{e e}^{2} / r_{D}^{2}}} \frac{1}{\left|\mathbf{r}_{1}-\mathbf{r}_{2}\right|}\left\{\exp \left[A_{e e}\left(\lambda_{e e}, r_{D}\right)\left|\mathbf{r}_{1}-\mathbf{r}_{2}\right|\right]\right. \\
& \left.-\exp \left[B_{e e}\left(\lambda_{e e}, r_{D}\right)\left|\mathbf{r}_{1}-\mathbf{r}_{2}\right|\right]\right\},
\end{aligned}
$$


where $\lambda_{\alpha \beta}\left(=\hbar / \sqrt{2 \pi \mu_{\alpha \beta} k_{B} T}\right)$ and $\mu_{\alpha \beta}$ are, respectively, the thermal de Broglie wavelength and reduced mass of the $\alpha-\beta$ pair, $k_{B}$ is the Boltzmann constant, $T$ is the plasma temperature, and the screening parameters $A_{\alpha \beta}$ and $B_{\alpha \beta}$ are given by $A_{\alpha \beta}^{2} \equiv\left(1-\sqrt{1-4 \lambda_{\alpha \beta}^{2} / r_{D}^{2}}\right) /\left(2 \lambda_{\alpha \beta}^{2}\right) \quad$ and $\quad B_{\alpha \beta}^{2}$ $\equiv\left(1+\sqrt{1-4 \lambda_{\alpha \beta}^{2} / r_{D}^{2}}\right) /\left(2 \lambda_{\alpha \beta}^{2}\right)$. After some mathematical manipulations, the equation of motion of the electron at $\mathbf{r}_{1}$ in strongly coupled semiclassical plasmas is then written as

$$
\begin{aligned}
\frac{d^{2} \mathbf{r}_{1}}{d t^{2}}= & -\frac{Z e^{2}}{m_{e} \sqrt{1-4 \lambda_{e i}^{2} / r_{D}^{2}}} \mathbf{r}_{1}\left\{\left[\frac{1}{r_{1}^{3}}+\frac{1}{r_{1}^{2}}\left(\frac{1-\sqrt{1-4 \lambda_{e i}^{2} / r_{D}^{2}}}{2 \lambda_{e i}^{2}}\right)^{1 / 2}\right] \exp \left[-\left(\frac{1-\sqrt{1-4 \lambda_{e i}^{2} / r_{D}^{2}}}{2 \lambda_{e i}^{2}}\right)^{1 / 2} r_{1}\right]\right. \\
& \left.-\left[\frac{1}{r_{1}^{3}}+\frac{1}{r_{1}^{2}}\left(\frac{1+\sqrt{1-4 \lambda_{e i}^{2} / r_{D}^{2}}}{2 \lambda_{e i}^{2}}\right)^{1 / 2}\right] \exp \left[-\left(\frac{1+\sqrt{1-4 \lambda_{e i}^{2} / r_{D}^{2}}}{2 \lambda_{e i}^{2}}\right)^{1 / 2} r_{1}\right]\right\} \\
& +\frac{e^{2}}{m_{e} \sqrt{1-4 \lambda_{e e}^{2} / r_{D}^{2}}} \mathbf{r}_{12}\left\{\left[\frac{1}{r_{12}^{3}}+\frac{1}{r_{12}^{2}}\left(\frac{1-\sqrt{1-4 \lambda_{e e}^{2} / r_{D}^{2}}}{2 \lambda_{e e}^{2}}\right)^{1 / 2}\right] \exp \left[-\left(\frac{1-\sqrt{1-4 \lambda_{e e}^{2} / r_{D}^{2}}}{2 \lambda_{e e}^{2}}\right)^{1 / 2} r_{12}\right]\right. \\
& \left.-\left[\frac{1}{r_{12}^{3}}+\frac{1}{r_{12}^{2}}\left(\frac{1+\sqrt{1-4 \lambda_{e e}^{2} / r_{D}^{2}}}{2 \lambda_{e e}^{2}}\right)^{1 / 2}\right] \exp \left[-\left(\frac{1+\sqrt{1-4 \lambda_{e e}^{2} / r_{D}^{2}}}{2 \lambda_{e e}^{2}}\right)^{1 / 2} r_{12}\right]\right\},
\end{aligned}
$$

where $m_{e}$ is the electron mass and $r_{12}\left(\equiv\left|\mathbf{r}_{1}-\mathbf{r}_{2}\right|\right)$ is the interelectronic distance. In the Wannier mode, ${ }^{3,14}$ the ejected two electrons would be placed at equal radial distances from the residual ion and also in opposite directions with respect to the residual ion in the ridge of the potential surface. Hence, the equation of motion in the Wannier configuration $\left[\mathbf{r}_{1}(\equiv \mathbf{r})=-\mathbf{r}_{2}\right]$ for the double-electron escape from the ion becomes

$$
\frac{d^{2} \mathbf{r}}{d t^{2}}=\frac{Z_{e}\left(r, \lambda, r_{D}, Z\right)}{m_{e}}\left[\frac{\partial}{\partial \mathbf{r}_{1}} V_{e i}\left(\mathbf{r}_{1}\right)\right]_{\mathbf{r}_{1}=\mathbf{r}},
$$

where $Z_{e}\left(r, \lambda, r_{D}, Z\right)$ represents the renormalized electron charge at the radial distance $r$ in strongly coupled semiclassical plasmas,

$$
Z_{e}\left(r, \lambda, r_{D}, Z\right)=-1+\frac{1}{4 Z} \frac{\eta\left(r, \lambda, r_{D}\right)}{\xi\left(r, \lambda, r_{D}\right)} .
$$

Here, $\eta\left(r, \lambda, r_{D}\right) / \xi\left(r, \lambda, r_{D}\right)$ term shows the correction due to the quantum and screening effects on the renormalized electron charge and $\eta\left(r, \lambda, r_{D}\right)$ and $\xi\left(r, \lambda, r_{D}\right)$ are, respectively,

$$
\begin{aligned}
\eta\left(r, \lambda, r_{D}\right)= & \frac{1}{\sqrt{1-4 \lambda^{2} / r_{D}^{2}}}\left\{\left[1+2\left(\frac{1-\sqrt{1-4 \lambda^{2} / r_{D}^{2}}}{2 \lambda^{2}}\right)^{1 / 2} r\right]\right. \\
& \times \exp \left[-2\left(\frac{1-\sqrt{1-4 \lambda^{2} / r_{D}^{2}}}{2 \lambda^{2}}\right)^{1 / 2} r\right] \\
& -\left[1+2\left(\frac{1+\sqrt{1-4 \lambda^{2} / r_{D}^{2}}}{2 \lambda^{2}}\right)^{1 / 2} r\right] \\
& \left.\times \exp \left[-2\left(\frac{1+\sqrt{1-4 \lambda^{2} / r_{D}^{2}}}{2 \lambda^{2}}\right)^{1 / 2} r\right]\right\},
\end{aligned}
$$

$$
\begin{aligned}
\xi\left(r, \lambda, r_{D}\right)= & \frac{1}{\sqrt{1-2 \lambda^{2} / r_{D}^{2}}}\left\{\left[1+\left(\frac{1-\sqrt{1-2 \lambda^{2} / r_{D}^{2}}}{\lambda^{2}}\right)^{1 / 2} r\right]\right. \\
& \times \exp \left[-\left(\frac{1-\sqrt{1-2 \lambda^{2} / r_{D}^{2}}}{\lambda^{2}}\right)^{1 / 2} r\right] \\
& -\left[1+\left(\frac{1+\sqrt{1-2 \lambda^{2} / r_{D}^{2}}}{\lambda^{2}}\right)^{1 / 2} r\right] \\
& \left.\times \exp \left[-\left(\frac{1+\sqrt{1-2 \lambda^{2} / r_{D}^{2}}}{\lambda^{2}}\right)^{1 / 2} r\right]\right\},
\end{aligned}
$$

where $\lambda\left(=\hbar / \sqrt{\pi m_{e} k_{B} T}\right)$ is the thermal de Broglie wavelength of the electron-electron pair. According to Wannier's configuration of ridge propagation, ${ }^{3}$ the escaping two electrons move independently with the screened renormalized electron charge $Z_{e}\left(r, \lambda, r_{D}, Z\right)$ in the field of the residual ion with the effective change $Z_{\text {eff }}\left(r, \lambda, r_{D}, Z\right)$ $\left[=-Z_{e}\left(r, \lambda, r_{D}, Z\right) Z\right]$ in strongly coupled semiclassical plasmas. If the quantum effects are absent $(\lambda \rightarrow 0)$ in the plasma, the screening parameters turned out to be $A_{e i} \rightarrow 1 / r_{D}, A_{e e}$ $\rightarrow 1 / r_{D}, B_{e i} \rightarrow \infty$, and $B_{e e} \rightarrow \infty$, so that the renormalized electron charge becomes $Z_{e}^{\prime}\left(r, r_{D}, Z\right)=-1+\left(e^{-r / r_{D}} / 4 Z\right)(1$ $\left.+2 r / r_{D}\right) /\left(1+r / r_{D}\right)$, i.e., the case of the weakly coupled plasma. ${ }^{15}$ From Eq. (6), we can show that the individual effective screened electron charge is given by $Z_{e} \rightarrow-1$ $+(1 / 4 Z)$ for the case of the free target atom or ion, i.e., $\lambda$ $\rightarrow 0$ and $r_{D} \rightarrow \infty$. In addition, the radial electron correlation effect on the renormalized electron charge would be neglected with increasing $Z$ so that the effective charge becomes $Z_{e} \rightarrow-1$. According to Wannier's work, it has been shown that the energy $(E)$ dependence of the cross section $(\sigma)$ near the ionization threshold $I$ for a free hydrogenlike atom or ion with nuclear charge $Z e$ is represented by a power law $\sigma \propto(E-I)^{\zeta}$, where $\zeta(Z)\left(=(1 / 4)[(100 Z-9) /(4 Z-1)]^{1 / 2}\right.$ $-1 / 4)$ is the Wannier exponent ${ }^{1,14}$ for the double-electron 


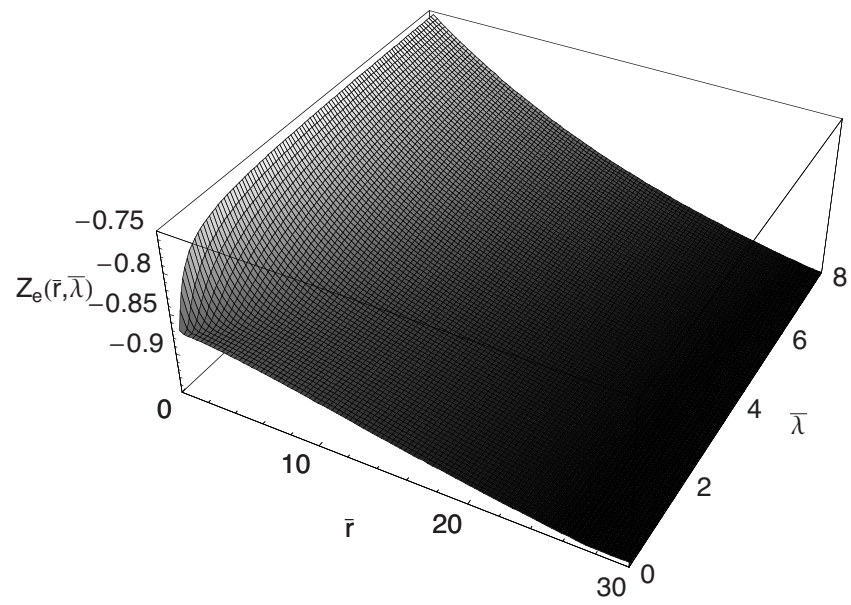

FIG. 1. The three-dimensional plot of the renormalized electron charge $Z_{e}$ as a function of the scaled de Broglie wavelength $\bar{\lambda}$ and scaled distance $\bar{r}$ when $Z=2$ and $\bar{r}_{D}=20$.

escape. For a pure Coulomb case, the solution of Eq. (5) is known as $r \propto t^{2 / 3} .{ }^{14}$ However, for the pseudopotential case in plasmas it is expected that the screened effective charge provides the detailed information on the properties of plasmas as well as the ionization mechanism. Hence, in strongly coupled semiclassical plasmas, however, the effective charge, $Z-1 / 4$, of the residual ion would be replaced by the screened effective charge $Z_{\text {eff }}\left(r, \lambda, r_{D}, Z\right)$ due to the influence of the quantum and screening effects. Therefore, the screened Wannier exponent function $\zeta\left(r, \lambda, r_{D}, Z\right)$ in strongly coupled semiclassical plasmas becomes

$$
\begin{aligned}
& \zeta\left(r, \lambda, r_{D}, Z\right) \\
& \quad=\frac{1}{4}\left\{\left[\frac{100 Z+16-25 \eta\left(r, \lambda, r_{D}\right) / \xi\left(r, \lambda, r_{D}\right)}{4 Z-\eta\left(r, \lambda, r_{D}\right) / \xi\left(r, \lambda, r_{D}\right)}\right]^{1 / 2}-1\right\} .
\end{aligned}
$$

The range of radial distances of importance for the Hamiltonian matrix element for the atomic ionization process is known as $r \approx a_{Z}{ }^{16}$ i.e., the distance between the escaping electron and residual ion is comparable to the Bohr radius, where $a_{Z}\left(=a_{0} / Z\right)$ is the Bohr radius of the hydrogenic ion with nuclear charge $Z e$ and $a_{0}\left(=\hbar^{2} / m_{e} e^{2}\right)$ is the Bohr radius of the hydrogen atom. The screened Wannier exponent for the double-electron escape from the target ion with nuclear charge $Z e$ in strongly coupled semiclassical plasmas is then obtained by the relation $\bar{\zeta}\left(\bar{\lambda}, \bar{r}_{D}, Z\right) \quad\left[=\left.\zeta\left(r, \lambda, r_{D}, Z\right)\right|_{r=a_{Z}}\right]$, where $\bar{\lambda}\left(\equiv \lambda / a_{Z}\right)$ is the scaled de Broglie wavelength and $\bar{r}_{D}$ $\left(\equiv r_{D} / a_{Z}\right)$ is the scaled Debye length.

Figure 1 represents the three-dimensional plot of the renormalized electron charge $Z_{e}$ for the double-electron escape from the ion in strongly coupled semiclassical plasmas as a function of the scaled de Broglie wavelength $\bar{\lambda}$ and scaled radial distance $\bar{r}\left(\equiv r / a_{Z}\right)$. As it is seen, the renormalized electron charge significantly increases with an increase in the de Broglie wavelength, especially for small radial distances. Figure 2 shows the renormalized electron charge $Z_{e}$ as a function of the scaled radial distance $\bar{r}$ for various values of the de Broglie wavelength. From this figure, it is found that the quantum effects on the renormalized electron charge

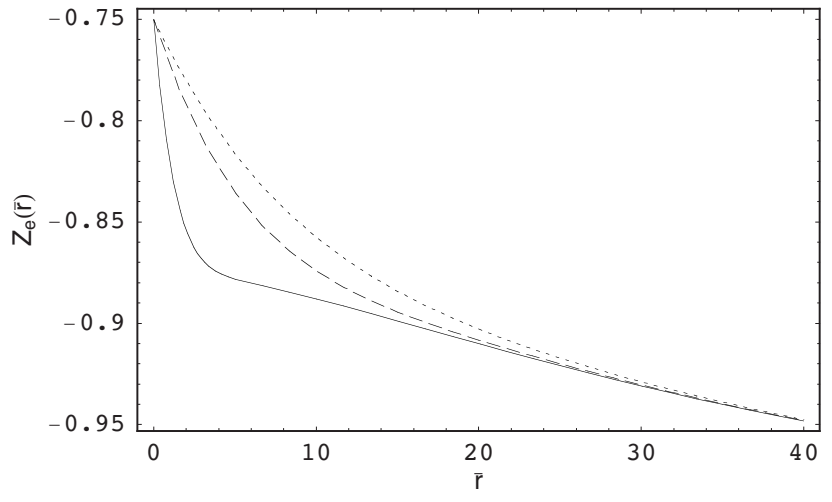

FIG. 2. The renormalized electron charge $Z_{e}$ as a function of the scaled distance $\bar{r}$ when $Z=2$ and $\bar{r}_{D}=30$. The solid line represents the case of $\bar{\lambda}$ $=1$. The dashed line represents the case of $\bar{\lambda}=4$. The dotted line represents the case of $\bar{\lambda}=6$.

decrease with increasing the radial distance beyond the Debye radius. Figure 3 represents the three-dimensional figure of the Wannier exponent $\bar{\zeta}$ as a function of the scaled Debye length $\bar{r}_{D}$ and scaled de Broglie wavelength $\bar{\lambda}$. As it is seen, the quantum effects enhance the Wannier exponent for the double-electron escape. It is also found that the quantum effect on the Wannier threshold law is more important than the plasma screening effect in strongly coupled semiclassical plasmas since the reaction zone is placed inside the Debye sphere. Hence, the threshold cross sections for the doubleelectron escape from the ion in strongly coupled semiclassical plasmas are expected to be smaller to those in classical plasmas since the screened Wannier exponent can be written as $\bar{\zeta}[=\partial \ln \sigma / \partial \ln (E-I)]$. Hence, we have found that the quantum effect plays an important role on the Wannier threshold law for the double-electron escape in strongly coupled semiclassical plasmas. These results would provide useful information on the quantum and plasma screening effects on the threshold behavior of the double-electron escape from the ion in strongly coupled semiclassical plasmas.

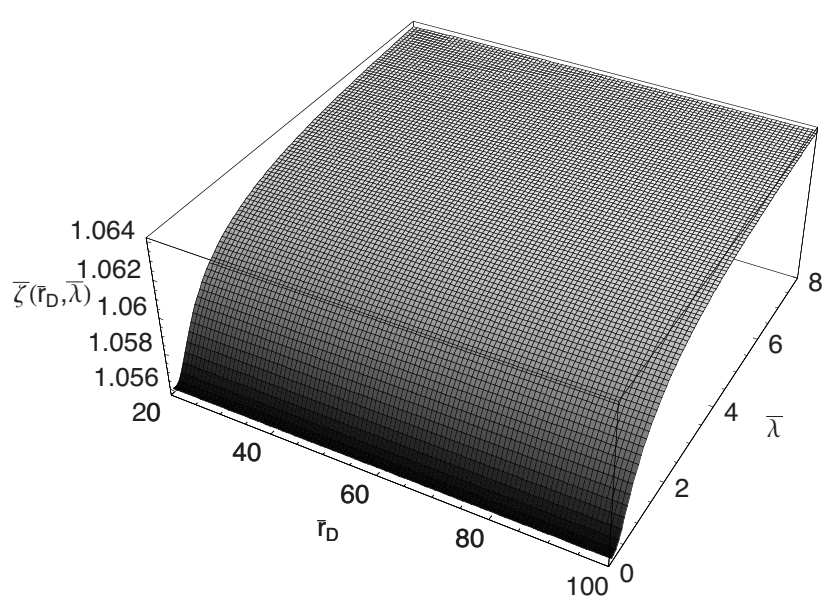

FIG. 3. The three-dimensional plot of the Wannier exponent $\bar{\zeta}$ as a function of the scaled Debye length $\bar{r}_{D}$ and scaled de Broglie wavelength $\bar{\lambda}$ when $Z=2$. 
One of the authors (Y.-D.J.) gratefully acknowledges Director-General Professor O. Motojima, Director Professor M. Sato, Director Professor Y. Hirooka, and Professor I. Murakami for warm hospitality while visiting the National Institute for Fusion Science (NIFS) in Japan as a long-term visiting professor. The authors are also grateful to NIFS for supporting the research. This work was done while Y.-D.J. visited NIFS.

${ }^{1}$ G. H. Wannier, Phys. Rev. 90, 817 (1953).

${ }^{2}$ D. Kato and S. Watanabe, Phys. Rev. A 56, 3687 (1997).

${ }^{3}$ J. Berakdar, Concepts of Highly Excited Electronic Systems (Wiley, Weinheim, 2003), p. 79.

${ }^{4}$ V. P. Shevelko, Atoms and Their Spectroscopic Properties (Springer, Berlin, 1997), p. 141.

${ }^{5} \mathrm{~S}$. P. Khare, Introduction to the Theory of Collisions of Electrons with Atoms and Molecules (Kluwer, New York, 2002), p. 125.
${ }^{6}$ V. P. Shevelko, H. Tawara, F. Scheuermann, B. Fabian, A. Müller, and E. Salzborn, J. Phys. B 38, 525 (2005).

${ }^{7}$ A. Müller, Adv. At., Mol., Opt. Phys. 55, 293 (2008).

${ }^{8}$ F. B. Baimbetov, Kh. T. Nurekenov, and T. S. Ramazanov, Phys. Lett. A 202, 211 (1995).

${ }^{9}$ T. S. Ramazanov and S. K. Kodanova, Phys. Plasmas 8, 5049 (2001).

${ }^{10}$ T. S. Ramazanov and K. N. Dzhumagulova, Phys. Plasmas 9, 3758 (2002).

${ }^{11}$ T. Ramazanov, K. Galiyev, K. N. Dzhumagulova, G. Röpke, and R. Redmer, Contrib. Plasma Phys. 43, 39 (2003).

${ }^{12}$ T. S. Ramazanov, K. N. Dzhumagulova, and Y. A. Omarbakiyeva, Phys. Plasmas 12, 092702 (2005).

${ }^{13}$ T. S. Ramazanov and K. N. Turekhanova, Phys. Plasmas 12, 102502 (2005).

${ }^{14}$ G. F. Drukarev, Collisions of Electrons with Atoms and Molecules (Plenum, New York, 1987), p. 180.

${ }^{15}$ Y.-D. Jung, Phys. Scr. 72, 237 (2005).

${ }^{16} \mathrm{H}$. A. Bethe and E. E. Salpeter, Quantum Mechanics of One- and TwoElectron Atoms (Springer-Verlag, Berlin, 1957), p. 316. 